\title{
Diagnóstico e prevalência de sarcopenia em idosos institucionalizados do município de João Pessoa-PB
}

\author{
Janilson Avelino da Silva \\ Graduado em Nutrição pela Universidade Federal da Paraíba-UFPB \\ Doutor em Ciências da Nutrição pela UFPB \\ Professor do curso de Nutrição do Centro Universitário-Uniesp, João Pessoa-PB

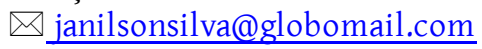

\section{Aléssio Tony Cavalcanti de Almeida}

Graduado em Economia pela UFPB e em Sistemas em Telecomunicações pelo Instituto Federal de Tecnologia da Paraíba-IFPB. Doutor em Economia aplicada pelo Programa de Pós-Graduação em Economia. Professor

Adjunto III do Departamento de Economia da UFPB, João Pessoa-PB

ه $\underline{\text { alessiotony@gmail.com }}$

Ithaissa Lacerda Tavares

Graduada em Nutrição pela UFPB. Nutricionista Clínica

$\triangle$ ithaissa.bsf@hotmail.com

Keyth Sulamitta de Lima Guimarães

Nutricionista e Enfermeira pela UFPB

Doutora em Ciências da Nutrição pela UFPB

Professora de Enfermagem e Nutrição do Unipê, João Pessoa-PB

$\bowtie$ keyth.sulamitta.lima@gmail.com

Maria José de Carvalho Costa

Graduada em Nutrição pela Universidade Federal de Pernambuco-UFPE.

Doutora em Nutrition Et Alimentation - Université de Bourgogne, França.

Professora Associada da UFPB, João Pessoa-PB

凶. $\underline{\text { mjc.costa@terra.com.br }}$

Renata Leite Tavares

Graduada em Nutrição pela UFPB

Doutoranda em Ciências da Nutrição pela UFPB

Professora do curso de Nutrição e Educação Física do IESP

凶renataltav@gmail.com

\section{Sandyerlly da Paz do Nascimento}

Graduada em Nutrição pela UFPB Nutricionista Clínica

هsandyerlly@hotmail.com

\section{Maria da Conceição Rodrigues Gonçalves}

Graduada em Nutrição pela UFPB. Doutora em Produtos Naturais e Sintéticos Bioativos pela UFPB

Professora Titular da UFPB, João Pessoa-PB

$\triangle$ mariadaconceicaorgoncalves@gmail.com 


\title{
Resumo:
}

A sarcopenia é uma doença muscular que se desenvolve comumente de forma progressiva e crônica. Ela possui relação com a perda da funcionalidade, com a incapacidade, redução da independência, aumento da fragilidade, mau prognóstico da saúde do idoso e mortalidade, pode ndo gerar altos custos pessoais, sociais e para o sistema de saúde. O objetivo desse estudo é verificar a prevalência de sarcopenia em idosos institucionalizados no município de João Pessoa-PB. Trata-se de um estudo epidemiológico, observacional, analítico, transversal emque foramselecionados 100 idosos, $\geq 60$ anos, de cinco Instituições de Longa Permanência para Idosos por meio de cálculo amostral e randomização. Foram avaliados parâmetros sociodemográficos, clínicos, antropométricos, de retinolemia, de estresse oxidativo e inflamação. Os dados foram analisados por meio de uma regressão logística multivariada no software R. Adotou-se o p<0,05. A sarcopenia foi atestada usando-se o algoritmo do Grupo de Trabalho Europeu de sarcopenia em idosos de 2019. Encontrou-se uma prevalência de sarcopenia em $37 \%$ da amostra (37 idosos), sendo 36\% considerada severa. Observou-se que os indivíduos sarcopênicos geralmente são homens ( $p=0,00$; IC $95 \%=4,04-46,37$ ), possuem retinolemia baixa ( $p=0,01 ;$ IC $95 \%$ 0,04-0,63) e possuem excesso de peso ( $p=0,04$; IC 95\% 0,03-0,93), independente de outros fatores de risco. Conclusão: No presente estudo, observou-se que existe uma elevada prevalência de sarcopenia nesse grupo institucionalizado, quase $100 \%$ emalto grau, principalmente em homens com baixas quantidades de vitamina A e com excesso de peso.

Palavras-chave: Envelhecimento, Instituição de Longa Permanência para idosos, Força da mão, Composição corporal. Velocidade de caminhada.

\section{Diagnosis and prevalence of sarcopenia in institutionalized elderly in the city of João Pessoa-PB}

\begin{abstract}
:
Sarcopenia is a muscular disease that commonly develops in a progressive and chronic way. It is related to loss of functionality, disability, reduced independence, increased fragility, poor prognosis for the health of the elderly and mortality, which can generate high personal, social and health system costs. The objective of this study is to verify the prevalence of sarcopenia in institutionalized elderly in the city of João Pessoa-PB. This is an epidemiological, observational, analytical, cross-sectional study in which 100 elderly people, $\geq 60$ years old, fromfive Long Term Care Institutions for the Elderly were selected through sample calculation and randomization. Sociodemographic, clinical, anthropometric, retinolemia, oxidative stress and inflammation parameters were evaluated. The data were analyzed using multivariate logistic regression using the R software. $\mathrm{P}<0.05$ was adopted. Sarcopenia was attested using the European Working Group's sarcopenia algorithmin the elderly of 2019. A prevalence of sarcopenia was found in $37 \%$ of the sample (37 elderly), with $36 \%$ being considered severe. It was observed that sarcopenic individuals are usually men $(p=0.00 ; 95 \% C I=4.04$ 46.37), have low retinolemia ( $p=0.01 ; 95 \%$ CI 0.04-0.63 $)$ and are overweight $(p=0.04 ; 95 \%$ CI0.03-0.93), regardless of other risk factors. Conclusion: In the present study, it was observed that there is a high prevalence of sarcopenia in this institutionalized group, almost $100 \%$ in a high degree, especially in men with low amounts of vitamin A and overweight.
\end{abstract}

Keywords: : Aging, Long-Term Care Facility for the Elderly, Hand Strength, Body Composition. Walking speed. 


\title{
Diagnóstico y prevalencia de sarcopenia en ancianos institucionalizados en la ciudad de João Pessoa-PB
}

\begin{abstract}
Resumen:
La sarcopenia es una enfermedad muscular que comúnmente se desarrolla de forma progresiva y crónica. Está relacionado con la pérdida de funcionalidad, discapacidad, independencia reducida, fragilidad incrementada, mal pronóstico para la salud de los ancianos y mortalidad, lo que puede generar altos costos personales, sociales y del sistema de salud. El objetivo de este estudio es verificar la prevalencia de sarcopenia en ancianos institucionalizados en la ciudad de João Pessoa-PB. Este es un estudio epidemiológico, observacional, analítico y transversal en el que se seleccionaron 100 personas mayores, $\geq 60$ años de edad, de cinco instituciones de atención a largo plazo para ancianos mediante el cálculo de la muestra y la aleatorización. Se evaluaron parámetros sociodemográficos, clínicos, antropométricos, retinolemia, estrés oxidativo e inflamación. Los datos se analizaron mediante regresión logística multivariada utilizando el software R. Se adoptó $\mathrm{P}<0.05$. La sarcopenia se atestiguó utilizando el algoritmo de sarcopenia del Grupo de Trabajo Europeo en los ancianos de 2019. Se encontró una prevalencia de sarcopenia en el 37\% de la muestra (37 ancianos), con un 36\% considerado grave. Se observó que los individuos sarcopénicos suelen ser hombres $(p=0.00$; IC 95\% = 4.04-46.37), tienen baja retinolemia ( $p=0.01$; IC 95\% 0.04-0.63) y tienen sobrepeso ( $p=0.04$; IC 95\% 0.03-0.93), independientemente de otros factores de riesgo. Conclusión: En el presente estudio, se observó que existe una alta prevalencia de sarcopenia en este grupo institucionalizado, casi $100 \%$ en un alto grado, especialmente en hombres con bajas cantidades de vitamina A y sobrepeso.

Palabras clave: Envejecimiento, Centro de atención a largo plazo para ancianos, Fuerza de la mano, Composición corporal. La velocidad al caminar.
\end{abstract}

\section{INTRODUÇÃO}

O termo sarcopenia foi criado por Irwin Rosemberg em 1989 para se referir a perda de massa muscular associada à idade. Dados epidemiológicos mostram que a prevalência de sarcopenia em idosos institucionalizados pode variar entre 18-73\% (RODRÍGUEZ-REJÓN et al., 2019), isso podendo ser devido à ausência de um consenso universal para diagnóstico dessa síndrome, e pelo fato de coexistirem diversos instrumentos para esse diagnóstico. Os homens apresentam maior prevalência de sarcopenia do que as mulheres (BASTIAANSE et al., 2012; MESQUITA et al., 2017).

A sarcopenia foi recentemente caracterizada e reconhecida como uma doença muscular pela classificação internacional das doenças - CID 10 (SHAW; DENISON; COOPER, 2017). Ela se desenvolve de forma progressiva e crônica, o que permite estabelecer estratégias de cuidados preventivos e/ou que possam amenizar o problema (CRUZ-JENTOFT, 2019). Mas também pode se desenvolver de forma aguda, como por exemplo, durante uma internação ou em uma situação de repouso prolongado (MARZETTI et al., 2017). 
Marzetti et al. (2017) afirmam que após os 40 anos, adultos saudáveis perdem aproximadamente $8 \%$ da massa muscular a cada 10 anos, e entre 40-70 anos esse valor sobe para $24 \%$, que se acelera após os 70 anos de forma progressiva.

As vias fisiopatológicas da sarcopenia são complexas, mas sabe-se que podem ocorrer alterações internas, como aumento do processo inflamatório, redução dos hormônios anabólicos, haver o acúmulo de radicais livres, aumento de atividades apoptóticas e alterações externas, como em situações de desuso das estruturas corporais (imobilidade e inatividade física). Esse processo pode ainda acontecer devido a uma ingestão deficiente de energia, proteínas e outros nutrientes (BASTIAANSE et al., 2012). À nível celular ocorrem perdas na inervação e inadaptações nas proporções das unidades motoras lentas e rápidas, bem como redução da área de secção transversal das fibras musculares (CHOI, 2016).

De acordo com as novas diretrizes europeias para sarcopenia em idosos (Writing Group for the European Working Group on Sarcopenia in Older People 2 - EGWSOP2), a verificação da quantidade e qualidade muscular serve para confirmar o diagnóstico, enquanto que sua funcionalidade triará a gravidade da patologia (CRUZ-JENTOFT, 2019).

Existem muitos fatores de risco associados a sarcopenia que reduzem o anabolismo e que, além disso, podem aumentar o catabolismo, como por exemplo o envelhecimento, a desnutrição, o aumento do estresse oxidativo e a elevação da inflamação. E reconhecê-los, identificando-os de forma precoce pode-se ajudar na prevenção e/ou tratamento dessa síndrome (KIM et al., 2016; MARZETTI et al., 2017).

É sabido que a sarcopenia possui relação com perda da funcionalidade, com a incapacidade, redução da independência, aumento da fragilidade, mau prognóstico da saúde do idoso e mortalidade podendo gerar altos custos pessoais, sociais e para o sistema de saúde (BASTIAANSE et al., 2012; LANDI et al., 2012; MARZETTI et al., 2017; LIGUORI et al., 2018). Apesar de sua importância, essa condição é raramente avaliada na prática clínica (ARAUJO; LAUKKANEN, 2019).

A força muscular é um dos fatores avaliados para obtenção do diagnóstico de sarcopenia. Ela diminui de forma mais intensa que a massa muscular. É recomendado por Marzetti et al., (2017) que o parâmetro "força" deva ser o primeiro elemento a ser testado em ambiente clínico com o uso de um equipamento chamado dinamômetro, embora a associação 
com a massa muscular seja necessária para esse diagnóstico. A dinapenia é considerada o melhor preditor de mortalidade e incapacidade, em comparação à perda de massa muscular isoladamente (SOARES et al., 2017).

Para verificação da massa muscular, o uso de bioimpedância e outros instrumentos, como a absorciometria por raios x de dupla energia (DEXA), a ressonância magnética e a tomografia computadorizada - sendo estes três últimos, de mais difícil acesso - devem ser utilizados para confirmação do diagnóstico (MARZETTI et al., 2017).

Além disso, é importante saber a gravidade da sarcopenia, fazendo com que seja necessário avaliar a capacidade física. A velocidade de marcha é um método mais prático para a rotina de profissionais da saúde, sendo que a bateria curta de testes físicos (SPPB) é mais apropriada em situações de pesquisas (CRUZ-JENTOFT, 2019).

A verificação da massa muscular tem sido recentemente estudada por meio de equipamentos, como ultrassom e também tem sido utilizados biomarcadores de sarcopenia, porém esses últimos elementos ainda não tem conseguido destaque como indicadores confiáveis, dada a complexidade dessa entidade e heterogeneidade da população investigada (TRIANA, 2019). Sendo assim, o objetivo do presente estudo é verificar a prevalência de sarcopenia em idosos institucionalizados no município de João Pessoa-PB.

\section{METODOLOGIA}

Trata-se de um estudo epidemiológico, observacional, analítico, transversal, o que não permite estabelecer uma relação de causa-efeito, nem capaz de estabelecer a temporalidade, muito embora sejam importantes na verificação de fatores de risco e desfechos em estudo (MEDRONHO, 2009).

Essa investigação foi previamente submetida ao Comitê de Ética em Pesquisa do Centro de Ciências da Saúde/UFPB, estando de acordo com a Resolução nº 466/12 do Conselho Nacional de Saúde - CNS/MS. Assim, obteve aprovação sob o número de protocolo 009/17. 
Todos os participantes do estudo assinaram o Termo de Consentimento Livre e Esclarecido TCLE.

O estudo foi realizado com 100 idosos de cinco (05) instituições de longa permanência para idosos (ILPI), entidades civis de direito privado, beneficentes, sem fins lucrativos, localizadas na cidade João Pessoa-PB. Foram incluídos: idosos com idade $\geq 60$ anos, de ambos os sexos, que sejam residentes nas ILPI por um período de tempo maior ou igual a três meses e excluídos os idosos com distúrbios neuropsiquiátricos que inviabilizassem os testes, idosos que usassem algum implante metálico no corpo ou marcapasso, acamados, impossibilitados de andar, que utilizassem suplementos que continham vitamina A, os que estivessem em uso de anti-inflamatórios e os que não aceitaram realizar as punções sanguíneas.

Para o cálculo amostral, considerou-se um nível de confiança de 95\% ( $Z=1,96)$, erro amostral de 9\%, uma prevalência de 30\% sarcopenia (de acordo com os dados de um estudo piloto realizado um ano antes do estudo original com 30 idosos institucionalizados), obtendose um valor de 100 idosos para o tamanho amostral. Os idosos foram selecionados usando-se uma tabela de números aleatórios.

Foram verificadas questões clínicas, sociodemográficas, antropométricas, de composição corporal e variáveis relacionadas a sarcopenia. Para a mensuração do peso, utilizou-se uma balança de bioimpedância (BIA) tetrapolar InBody ${ }^{\circ}$ 120, com capacidade de até $250 \mathrm{~kg}$ e precisão de 100g, e que também fazia medições dos compartimentos corporais, seguindo todas as orientações do fabricante. Realizou-se a estimativa da altura pela altura do joelho, usando a fórmula de Chumlea, Roche e Steinbaugh (1985), de acordo com o sexo.

O indicador utilizado para avaliar o estado nutricional foi o Índice de Massa Corporal - IMC, em que se divide o peso corporal (em kg) pela altura (em metros) ao quadrado. No Brasil, o Ministério da Saúde preconiza a utilização dos pontos de corte propostos por NSI (1994) como referência na avaliação do IMC de idosos (baixo-peso IMC $<22 \mathrm{~kg} / \mathrm{m}^{2}$; eutrofia IMC entre 22 e $27 \mathrm{~kg} / \mathrm{m}^{2}$; e sobrepeso IMC > $27 \mathrm{~kg} / \mathrm{m}^{2}$ ), mas estudos de avaliação do estado nutricional envolvendo a população idosa consideram em sua maioria os valores recomendados pela WHO (1997); (baixo peso IMC $<18,5 \mathrm{~kg} / \mathrm{m}^{2}$; eutrofia IMC entre 18,5 e 24,9 $\mathrm{kg} / \mathrm{m}^{2}$; sobrepeso IMC entre 25 e $29,9 \mathrm{~kg} / \mathrm{m}^{2}$ e obesidade IMC $\geq 30 \mathrm{~kg} / \mathrm{m}^{2}$ ). Assim, optou-se usar os dois parâmetros. A obesidade sarcopênica foi diagnosticada quando identificou-se 
indivíduos com sarcopenia e acúmulo de gordura corporal, situação de coexistência de sarcopenia e obesidade (BAUMGARTNER, 2000).

A Circunferência da Panturrilha foi aferida e um valor inferior a 31 centímetros foi utilizado para indicar depleção de massa muscular (VELAS et al., 1999). A musculatura adutora do polegar-MAP foi avaliada de acordo com Lameu et al. (2004). As circunferências do braço$\mathrm{CB}$, muscular do braço- $\mathrm{CMB}$, área muscular do braço corrigida-AMBc e dobra cutânea triciptal-DCT foram verificadas de acordo com as recomendações de Mussoi (2015).

A circunferência da cintura (CC) foi medida no final da expiração normal de cada sujeito, com aproximação de $0,1 \mathrm{~cm}$ no ponto médio entre a extremidade inferior da $12^{\mathrm{a}}$ costela e a extremidade superior da crista ilíaca, usando uma fita métrica inelástica. A circunferência do quadril (CQ) foi medida na parte mais saliente dos quadris. A relação cintura quadril (RCQ) foi obtida dividindo-se a CC pela CQ. A relação cintura-estatura (RCE) foi obtida dividindo-se a cintura (em centímetros) pela altura (em metros). O ponto de corte usado foi 0,5 que tem sido utilizado em pesquisas nacionais e internacionais com idosos (MILAGRES et al., 2019; TAWFIK, 2018).

As amostras de sangue foram coletadas, após 12 horas de jejum nas próprias instituições em tubos de ensaio protegidos da luz e analisadas no início do estudo. Alíquotas de soro foram armazenadas a $-80^{\circ} \mathrm{C}$ e não foram descongeladas até serem analisadas.

As concentrações séricas de retinol foram determinadas por Cromatografia Líquida de Alta Performance (HPLC) utilizando a metodologia recomendada por Furr, Tanumihardj, Oslon (1992). O ponto de corte utilizado para concentrações inadequadas de retinol sérico é < 1,05 $\mu \mathrm{mol} / \mathrm{L}$ (WHO, 1996; IVACG, 2003).

Foi realizada a avaliação da glicemia de jejum e creatinina e análise da alfa 1 glicoproteína ácida (A1GPA), como marcador inflamatório, usando-se kits comerciais Labtest seguindo as recomendações do fabricante. Para tais, foi utilizado o analisador automático Labmax 240 premium (Labtest, Minas Gerais, Brasil).

Como forma de verificação dos marcadores de estresse oxidativo, mediu-se a Capacidade Antioxidante Total - CAT pelo método descrito por Brand-Williams, Cuvelier e 
Berset (1995). Os resultados foram expressos como percentual da atividade antioxidante (AOA).

Verificou-se o Malondialdeído - MDA como forma de verificação da atividade oxidante por meio da reação do ácido tiobarbitúrico - TBARS, conforme método descrito por Ohkawa, Ohishi e Yagi (1979).

A sarcopenia foi atestada usando-se o algoritmo do Grupo de Trabalho Europeu de sarcopenia em idosos de 2019. Sabe-se que esta pode ser medida por meio da quantificação da força, da massa muscular e da capacidade física. A orientação é que se investigue uma sarcopenia provável, medindo-se a força, e em seguida, avalie-se a massa muscular; e que essa avaliação em conjunto, em caso de redução, confirma-se a sarcopenia. A verificação da capacidade física é descrita como uma forma de identificação da gravidade dessa patologia (CRUZ-JENTOFT et al., 2019).

Sendo assim, inicialmente, verificou-se a força, expressa pela Força de Preensão Manual - FPM. Para esta aferição foi utilizado o dinamômetro hidráulico Jamar em kg. As avaliações de FPM foram desenvolvidas de acordo com as orientações da American Society of Hand Therapists - ASHT (DESROSIERS et al., 1995). O idoso foi posicionado sentado e sem apoio de braço, com os ombros aduzidos e em rotação neutra. O cotovelo foi flexionado a $90^{\circ}$, com antebraço em posição neutra e punho variando de 0 a $30^{\circ}$ de extensão. Os pontos de corte utilizados para sarcopenia foram $<27 \mathrm{Kg}$ para homens e $<16 \mathrm{Kg}$ para as mulheres (DODDS et al., 2014).

Em seguida, procedeu-se com a obtenção das variáveis da bioimpedância (massa muscular dos membros superiores e inferiores), obtendo-se a Massa Muscular Apendicular MMA, somando-se a massa muscular dos braços e pernas. Foram utilizados os pontos de corte de Studenski (2014) (homens $<20$ e mulheres $<15 \mathrm{Kg} / \mathrm{m}^{2}$ ).

Além disso, avaliou-se a capacidade física, por meio da Short Physical Performance Battery (SPPB). Esse método abrange três domínios: testes de equilíbrio (pés juntos, calcanhar de um pé no outro pé e calcanhar de um pé diretamente a frente e tocando o outro pé, por 10 segundos cada), velocidade de marcha (caminhada em ritmo habitual cronometrada por 6 metros) e o teste de levantar e sentar da cadeira repetidas vezes (cinco vezes com os braços cruzados na frente do corpo), cada teste variando escores de 0 a 4 pontos, obtendo-se no 
máximo, 12 pontos, numa escala de 0-12, conforme Nakano (2011). O ponto de corte utilizado foi $\leq 8$ pontos para indivíduos com sarcopenia severa (CRUZ-JENTOFT et al., 2019).

Os dados foram apresentados em medidas de tendência central (média) e de dispersão (desvio padrão da média) para as variáveis contínuas e frequência e percentual, para as variáveis categóricas. Utilizou-se o teste $t$ de student para comparação de medidas contínuas, enquanto que o teste qui-quadrado para comparar dados categóricos entre grupos com e sem sarcopenia.

As associações entre sarcopenia (variável dependente) e covariáveis (variáveis independentes) foram estimadas através de odds ratios (OR) e 95\% do intervalo de confiança (IC) em um modelo de regressão logística multivariada. Valores de p inferiores a 0,05 foram considerados estatisticamente significantes. Todas as análises estatísticas foram realizadas usando o software R.

\section{RESULTADOS}

Foram avaliados 100 idosos institucionalizados, com $79 \pm 8$ anos, portadores de doenças crônicas não-transmissíveis-DCNT (Hipertensão arterial sistêmica (64\%), diabetes mellitus (31\%), dislipidemias (17\%), Alzheimer (13\%), Mal de Parkinson (19\%), depressão (26\%), ansiedade (27\%) e esquizofrenia (28\%), que vivem nessas instituições por um período médio de seis anos e meio. Os grupos (sarcopênicos e não sarcopênicos) não diferiram em relação ao número de doenças (Tabela 1).

Em relação aos parâmetros antropométricos, os idosos sarcopênicos possuem um índice de massa corporal - IMC menor $\left(23,00 \pm 4,18 \mathrm{~kg} / \mathrm{m}^{2}\right)$ em relação aos não sarcopênicos $\left(25,46 \pm 5,00 \mathrm{~kg} / \mathrm{m}^{2}\right)(\mathrm{p}=0,00)$; circunferência do braço - CB mais baixa $(25,44 \pm 2,81$ e 27,08 $\pm 4,5$ $\mathrm{cm}$, respectivamente; $p=0,04)$ e dobra cutânea triciptal - DCT menor $(15,00 \pm 6,23$ e 20,32 $\pm 9,00$ $\mathrm{mm}$, respectivamente; $\mathrm{p}=0,00$ ) em relação aos idosos não sarcopênicos.

Tabela 1 - Características clínicas e sociodemográficas dos idosos institucionalizados do município de João Pessoa-PB ( $\mathrm{n}=100), 2019$. 


\begin{tabular}{|c|c|c|c|c|c|}
\hline \multirow{2}{*}{$\begin{array}{c}\text { VARIÁVEL } \\
\text { Sexo }\end{array}$} & \multicolumn{2}{|c|}{$\begin{array}{l}\text { SARCOPÊNICO } \\
(\mathrm{n}=37)\end{array}$} & \multicolumn{2}{|c|}{$\begin{array}{c}\text { NÃO SARCOPÊNICO } \\
(n=63)\end{array}$} & \multirow[t]{2}{*}{$\begin{array}{c}\text { VALOR } \\
p\end{array}$} \\
\hline & $\mathrm{N}$ & $\%$ & $\mathrm{n}$ & $\%$ & \\
\hline Masculino & 29 & 29 & 5 & 05 & $0,00^{*}$ \\
\hline Feminino & 08 & 08 & 58 & 58 & \\
\hline \multicolumn{6}{|l|}{ Estado civil } \\
\hline Com companheiro & 06 & 06 & 08 & 08 & 0,62 \\
\hline Sem companheiro & 31 & 31 & 55 & 55 & \\
\hline \multicolumn{6}{|l|}{ Escolaridade } \\
\hline $\begin{array}{l}\text { Até fundamental } \\
\text { completo }\end{array}$ & 32 & 32 & 44 & 44 & 0,06 \\
\hline $\begin{array}{l}\text { Até ensino médio } \\
\text { completo }\end{array}$ & 05 & 05 & 19 & 19 & \\
\hline \multicolumn{6}{|l|}{$\begin{array}{c}\text { Ideia de } \\
\text { institucionalização }\end{array}$} \\
\hline Familiares & 20 & 20 & 30 & 30 & 0,28 \\
\hline Própria pessoa & 05 & 05 & 17 & 17 & \\
\hline $\begin{array}{c}\text { Outras } \\
\text { pessoas/instituições }\end{array}$ & 12 & 12 & 16 & 16 & \\
\hline \multicolumn{6}{|l|}{$\begin{array}{l}\text { Número de } \\
\text { medicamentos }\end{array}$} \\
\hline Até 4 medicamentos & 25 & 25 & 46 & 46 & 0,56 \\
\hline $\begin{array}{c}5 \text { ou mais } \\
\text { medicamentos }\end{array}$ & 12 & 12 & 17 & 17 & \\
\hline
\end{tabular}

${ }^{*} \mathrm{p}<0,05$ (teste Qui-quadrado de Pearson).

Fonte: Autor, 2019.

A prevalência de sarcopenia foi de $37 \%$ na amostra (37 idosos). Sendo que, $36 \%$ dos idosos encontram-se em sarcopenia severa (Figura 1). Os homens possuem menor força de preensão manual - FPM no grupo sarcopênico em detrimento dos não sarcopênicos $(14,0 \pm 6,0$ $\mathrm{kg}$ e $32,20 \pm 4,38 \mathrm{~kg}(\mathrm{p}=0,00)$, respectivamente). As mulheres sarcopênicas possuem menor peso $(45,10 \pm 7,3 \mathrm{~kg} ; \mathrm{p}=0,00), \operatorname{IMC}\left(20,43 \pm 40,1 \mathrm{~kg} / \mathrm{m}^{2} ; \mathrm{p}=0,00\right), \mathrm{CB}(23,00 \pm 2,8 \mathrm{~cm} ; \mathrm{p}=0,01)$, circunferência muscular do braço - $\mathrm{CMB}(17,00 \pm 2,6 \mathrm{~cm} ; \mathrm{p}=0,01)$, área muscular do braço corrigida - AMBc $\left(16,53 \pm 7,17 \mathrm{~cm}^{3} ; \mathrm{p}=0,02\right)$ e massa muscular - MM $(13,62 \pm 0,63 \mathrm{~kg} ; \mathrm{p}=0,00)$ 
quando comparadas as não sarcopênicas $(61,3 \pm 13 \mathrm{~kg}, 25,52 \pm 5,0,27,11 \pm 4,62 \mathrm{~cm}, 21,00 \pm 4,54$ $\mathrm{cm}, 30,00 \pm 16,00,19,19 \pm 3,20 \mathrm{~kg}$, respectivamente). 0 teste SPPB (capacidade física) obteve um valor médio de $3,33 \pm 2,65$ pontos.

Em relação a circunferência da cintura do grupo, observou-se que nos homens, 67,6\% ( $n=23)$ encontram-se em risco de doenças cardiometabólicas ( $n=34)$. Nas mulheres, 92,5 \% (n =61) encontram-se nessa mesa categoria de risco $(n=66)$, porém não houve diferença entre os grupos sarcopênicos e não sarcopênicos $(p>0,05)$.

A respeito do percentual de gordura, o valor médio de $36 \%$ de gordura corporal os colocam em um patamar de risco de doenças associadas a obesidade para toda amostra (100\%), sendo $76 \%$ dos idosos com elevado percentual de gordura corporal.

Figura 1: Algoritmo adaptado do $2^{\circ}$ Grupo de Trabalho Europeu sobre Sarcopenia em Idosos. Diagnóstico e gravidade de sarcopenia em idosos institucionalizados do município de João Pessoa-PB ( $\mathrm{n}=100), 2019$.

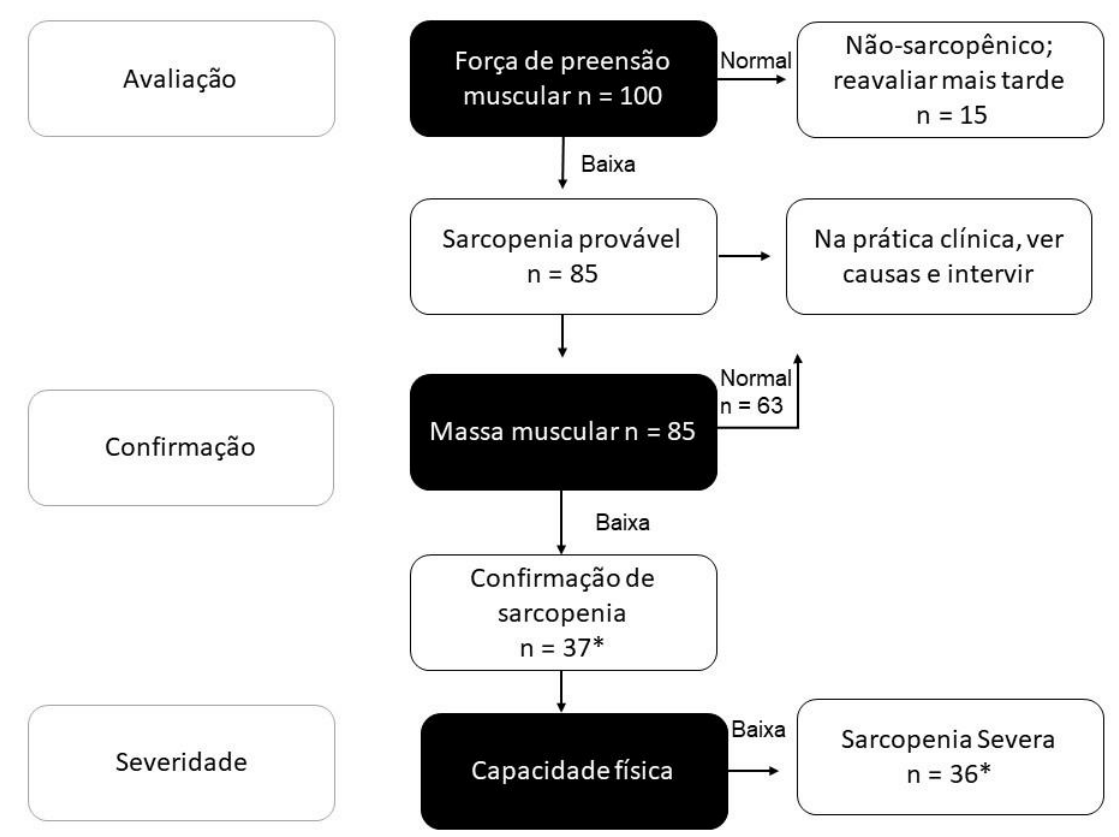

*Apenas um idoso possui sarcopenia de grau moderado.

Fonte: Autor, 2019. 
Após ajuste para os possíveis fatores de confusão, observou-se que os indivíduos sarcopênicos geralmente são homens $(p=0,00)$, possuem retinolemia baixa $(p=0,01)$ e detém excesso de peso $(p=0,04)$, independente de outros fatores de risco (Tabela 2 ).

A creatinina sérica demonstrou-se mais elevada no grupo sarcopênico $(0,86 \pm 0,2$ $\mathrm{mg} / \mathrm{dL})$ quando comparada ao grupo não sarcopênico $(0,77 \pm 0,2 \mathrm{mg} / \mathrm{dL})$. O retinol sérico no grupo com sarcopenia foi quantificado em $1,28 \pm 0,46 \mu \mathrm{mol} / \mathrm{L}$, enquanto que o grupo não

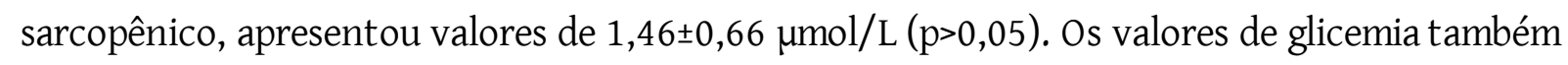
não diferiram entre os grupos $(p>0,05)$.

Tabela 2 - Regressão logística associando a sarcopenia a fatores de risco em instituições de longa permanência para idosos $(n=100)$. João Pessoa-PB, 2019.

\begin{tabular}{llll}
\hline VARIÁVEL & $\mathbf{p}$ & OR & $\mathbf{9 5 \%}$ IC \\
\hline Idade & 0,43 & 0,97 & $0,90-1,04$ \\
Sexo masculino & $0,00^{*}$ & 13,60 & $4,04-46,37$ \\
Número de doenças & 0,14 & 0,75 & $0,50-1,08$ \\
Período de institucionalização & 0,50 & 1,00 & $0,99-1,01$ \\
Escolaridade (até fundamental completo) & 0,70 & 0,75 & $0,17-3,22$ \\
Baixo peso & 0,20 & 2,46 & $0,64-10,48$ \\
Excesso de peso & $0,05^{*}$ & 0,20 & $0,03-0,93$ \\
Glicemia de jejum & 0,94 & 1,07 & $0,15-8,44$ \\
Vitamina A (retinol sérico) & $0,01^{*}$ & 0,19 & $0,04-0,63$ \\
Alfa glicoproteína ácida (AGP) & 0,57 & 0,10 & $0,96-1,01$ \\
Capacidade antioxidante total (CAT) & 0,54 & 0,10 & $0,95-1,02$ \\
Malondialdeído (MDA) & 0,91 & 1,03 & $0,58-1,85$ \\
Creatinina & 0,61 & 2,58 & $0,06-108,32$ \\
Musculatura adutora do polegar (MAP) & 0,10 & 0,10 & $0,79-1,25$ \\
\hline OR = odds ratio; 95\% IC = intervalo de confiança. & & & \\
& & & \\
\hline
\end{tabular}


Fonte: Autor, 2019.

\section{DISCUSSÃO}

O envelhecimento pode acarretar diversos problemas orgânicos para os idosos e um desses é a sarcopenia, considerada por Cruz-Jentoft et al. (2010) como uma síndrome geriátrica. Sendo assim, observa-se a necessidade de avaliação de fatores de risco e sua relação com a incapacidade funcional, fragilidade e mortalidade. Isso fica mais evidente nos idosos institucionalizados, que nem sempre possuem cuidados personalizados que correspondam às suas necessidades (BAIERLE et al., 2015; PINHEIRO et al., 2016).

Os idosos do presente estudo vivem por um período médio (seis anos e meio) similar ao estudo de Rosa, Moraes e Filha (2016) e possuem, em sua maioria, hipertensão arterial sistêmica, como na investigação de Ongan e Rakıcıo`lu (2015).

A feminização da velhice é destacada na presente investigação, pois a maioria da amostra é composta por mulheres ( $\mathrm{n}=66$ ), corroborando com outros achados (CARNEY, 2018; OLAWUYI; ADEOYE, 2018).

A maior parte da amostra possui 79 anos de idade, e idosos considerados na "quarta idade" tem sido o grupo que mais cresce no Brasil e no mundo, refletindo-se nessa investigação (MINAYO; FIRMO, 2019). A idade avançada é um fator de risco para essa patologia e a sarcopenia acomete idosos geralmente na faixa etária média do atual estudo (MARTY et al., 2017).

Encontrou-se uma prevalência de sarcopenia em 37\% da amostra (36\% severa), divergindo do estudo de Rodríguez-Rejón; Ruiz-López e Artacho (2019) que a quantificou em mais da metade da amostra institucionalizada $(60,1 \%) ; 58,1 \%$ com sarcopenia severa. Um estudo de Bravo-José et al. (2018) encontrou prevalência de 41,1\%, estando estes valores bem aproximados ao do presente estudo, também em idosos institucionalizados, tendo um percentual de $66 \%$ de sarcopenia severa.

Os pontos de corte para diagnóstico de sarcopenia do Consenso Europeu mudaram recentemente - 2019, comparados ao do consenso de 2010. Rodríguez-Rejón; Ruiz-López e 
Artacho (2019), ao compararem os dois consensos, observaram que os valores da prevalência e de gravidade de sarcopenia se mostraram praticamente iguais $(p>0,05)$. Um dos pontos fortes desse segundo consenso é o fato de terem considerado dados dos estudos com idosos institucionalizados, validando sua utilização para esse público (RODRIGUEZ-REJÓN; RUIZLÓPES; ARTACHO, 2019).

$\mathrm{Na}$ atual investigação, ser homem é fator de risco independente de outros fatores para a sarcopenia (OR 13,6, 95\% IC 4,04-46,37), constatando-se, coincidentemente, a mesma razão de chance, de treze vezes maior, no estudo de Landi et al. (2012) em uma população institucionalizada ( $n=122)$ com faixa etária similar ao dos idosos do presente estudo e também descrita por Bastiaanse et al. (2012). Os homens geralmente possuem uma maior perda de massa muscular em relação as mulheres com o aumento da idade que pode estar associada a redução da testosterona e ao hormônio do crescimento (SANTOS et al., 2015). Entretanto, no estudo de Bravo-José et al. (2018) a prevalência de sarcopenia foi maior nas mulheres.

O estado nutricional pode ser um fator determinante para o desenvolvimento da sarcopenia. No presente estudo, observou-se que idosos com excesso de peso possuem maior risco de serem sarcopênicos (OR 0,2 95\% IC 0,03-0,93). Tal constatação foi descrita, como sendo um fator de risco para a sarcopenia, por Kim et al. (2016), mesmo que Sieber (2019) tenha relatado que a desnutrição é um fator de risco preponderante no desenvolvimento da sarcopenia. Vlietstra et al. (2019) encontraram uma relação significativa entre elevado percentual de gordura e sarcopenia ao investigar pacientes com osteoartrite e artrite reumatoide.

Em relação a classificação do índice de massa corporal - IMC dos sarcopênicos, 11\% exibiu excesso de peso pela referência da NSI (1994), valor esse aumentado para 30\% quando utilizados os pontos de corte da WHO (1997).

É descrito na literatura que o aumento do IMC está associado a menores concentrações de retinol sérico e que a vitamina A pode estar envolvida nos processos de regulação de gordura corporal, principalmente na presença da deficiência de vitamina A. Tal fato é validado por estudos comparativos entre indivíduos obesos e eutróficos em que ambos exibem ingestão de vitamina A adequada, mas que os primeiros possuem redução de vitamina A sérica (BENTO et al., 2018). 
De acordo com os dados da atual investigação, considerou-se os idosos como obesos sarcopênicos, visto que tal fenômeno é descrito por Cruz-Jentoft et al. (2019), como sendo uma condição que envolve a redução da massa muscular no contexto de um acúmulo de gordura corporal, resultados esses encontrados em grande parte da amostra (76\% possuem elevado percentual de gordura e 100\% encontram-se na faixa de risco de doenças associadas a obesidade) (JACKSON; POLLOCK, 1978; JACKSON; POLLOCK; WARD, 1980; LOHMAN, 1988). HSU et al. (2019) afirmam que existe um maior risco de doenças metabólicas e maior incapacidade em obesos sarcopênicos do que pessoas com sarcopenia ou obesidade sozinhas.

Nesse contexto, sabe-se que a obesidade exacerba a sarcopenia, aumentando a infiltração de gordura no músculo, diminuindo a função física e aumentando o risco de mortalidade (CRUZ-JENTOFT et al., 2019; MARTY et al., 2017).

Pode existir também uma interação entre obesidade e sarcopenia, de modo que a perda de massa muscular pode gerar inatividade física, que pode levar a um menor gasto energético e, consequentemente haver o aumento do risco de obesidade, do mesmo modo que o acúmulo de gordura pode gerar um processo inflamatório que pode se refletir em sarcopenia (CHOI, 2016).

Parâmetros antropométricos relacionados a composição corporal podem ser considerados medidas em uma avaliação inicial, possuindo fácil utilização e como teste de triagem rápida e de baixo custo para sarcopenia em ILPI, principalmente quando outros instrumentos e métodos não estão disponíveis, fato não incomum na atenção primária à saúde - APS (LANDI et al., 2012; TOSATO et al., 2017). Observou-se no atual estudo que a redução desses parâmetros foi mais exibida nas mulheres do que nos homens. Resultados condizentes com um estudo realizado com 173 mulheres idosas, mostrando que os indicadores antropométricos de área muscular do braço corrigida e circunferência da panturrilha estão intimamente relacionados com sarcopenia (PINHEIRO et al., 2019).

Apesar de ser descrito na literatura que o envelhecimento pode levar os indivíduos idosos a um processo de inflamação crônica de baixo grau e elevação do estresse oxidativo (MARZETTI etal., 2017; CHOI, 2016), na atual investigação não se observou diferença (p>0,05) entre a alfa glicoproteína ácida - AGP entre idosos com ou sem sarcopenia, bem como nos 
parâmetros de estresse oxidativo, como capacidade antioxidante total - CAT e malondialdeído - MDA.

Como postula-se que a sarcopenia pode estar ligada a alterações no estresse oxidativo, decidiu-se avaliar a vitamina A por ser uma importante vitamina antioxidante, com funções clássicas bem definidas, porém suas relações com a capacidade física e sarcopenia ainda não foram bem exploradas, principalmente em idosos institucionalizados.

Pesquisas anteriores, com idosos mostraram que algumas vitaminas e antioxidantes plasmáticos, quando reduzidos, incluindo a vitamina A, estão associadas a limitações físicas e a incapacidade física, fatores de análise importantes para diagnóstico da sarcopenia, conforme atestado por Cesari et al. (2004), Lauretani et al. (2008) e Welch et al. (2020). O presente estudo reforça esses achados, visto que conter concentrações de retinol sérico mais reduzidas estão associadas, independente de outros fatores de risco, a sarcopenia (OR 0,19 95\% IC 0,04-0,63).

Quanto a creatinina sérica, sabe-se que é um produto do metabolismo da fosfocreatina muscular, elemento proporcional a massa muscular, indicador da reserva da musculatura e considerado como biomarcador de sarcopenia e que a sua elevação está associada a perdas musculares, sendo assim, os achados do presente estudo corroboram com tal informação, haja vista essa variável estar elevada em indivíduos sarcopênicos $(p<0,05)$. Resultados similares foram demonstrados por Fried et al. (2007) em um estudo longitudinal com 3026 idosos não institucionalizados com mais de 70 anos de idade (TOSATO et al., 2017).

Algumas limitações existiram na presente investigação, como o desenho do estudo que não permite estabelecer relações causais, entretanto as informações podem servir de base para estudos longitudinais e de intervenções. Além disso, não foi possível avaliar mais variáveis que pudessem influenciar esses resultados, visto a necessidade de dispêndio de muito mais tempo por parte dos idosos institucionalizados, que rotineiramente não encontram-se muito disponíveis para contribuir com as pesquisas.

É importante compreender os processos responsáveis pela redução da capacidade de execução das atividades de vida diária - AVD associados à idade em idosos institucionalizados para desenvolver estratégias para prevenir e/ou retardar a incapacidade e a morbimortalidade associada entre os idosos. 


\section{CONCLUSÃO}

No presente estudo, observou-se de forma inédita que existe uma elevada prevalência de sarcopenia nesse grupo institucionalizado, sendo quase $100 \%$ em alto grau, principalmente em homens com baixas quantidades de vitamina A e com excesso de peso, fatores de risco independentes atestados pela análise de regressão. Sugere-se que as medidas de controle dessa doença não foram efetivas, visto essa gravidade em quase toda a amostra.

Recomenda-se assim, uma assistência nutricional e de atividade física mais focadas nesses fatores de risco que podem estar associados a sarcopenia, sabendo-se que não existe uma sistematização dessa última prática nessas instituições. Assim, este estudo pode contribuir no sentido de alertar os gestores locais, municipais e de outras regiões do Brasil sobre a importância do cuidado integral, de qualidade e interprofissional para com essa população, que normalmente é esquecida pelo poder público.

Destarte, espera-se que esses resultados possam também orientar decisões clínicas e possam contribuir com o desenvolvimento de políticas públicas para a população idosa institucionalizada. Intervenções precoces são necessárias para que os idosos identificados como tendo maior risco de sarcopenia possam evitar suas consequências adversas à saúde.

Mais estudos são necessários para elucidar as estratégias necessárias para reverter esse quadro quando a sarcopenia ainda não está avançada, bem como encontrar maneiras de prevenção aplicadas aos idosos institucionalizados.

\section{REFERÊNCIAS}

ARAUJO, Claudio Gil; LAUKKANEN, Jari Antero. Músculo Cardíaco e Músculo Esquelético Conectados pelo Sistema Nervoso Autônomo. Arquivos Brasileiros de Cardiologia, São Paulo , v. 112, n. 6, p. 747-748, 2019. Disponível em: < http://www.scielo.br/pdf/abc/v112n6/pt_0066-782X-abc-112-06-0747.pdf>. Acesso em 05 Jan. 2019. 
BAIERLE, Marília; NASCIMENTO, Sabrina N.; MORO, Angela M.; BRUCKER, Natália; FREITAS, Fernando; GAUER, Bruna; DURGANTE, Juliano; BORDIGNON, Suelen; ZIBETTI, Murilo; TRENTINI, Clarissa M.; DUARTE, Marta M. M. F.; GRUNE, Tilman; BREUSING, Nicolle; GARCIA, Solange C. Relationship between Inflammation and Oxidative Stress and Cognitive Decline in the Institutionalized Elderly. Oxidative Medicine and Cellular Longevity, Porto Alegre, $\quad$ v. $\quad 2015, \quad$ p. $\quad 1-12, \quad 2015 . \quad$ Disponível $\quad$ em: http://downloads.hindawi.com/journals/omcl/2015/804198.pdf>. Acesso em 05 Jan. 2019.

BASTIAANSE, Luc P.; HILGENKAMP, T. I., ECHTELD, M.A., EVENHUIS, H.M. Prevalence and associated factors of sarcopenia in older adults with intellectual disabilities. Research in Developmental Disabilities, v. 33, n.06, p. 2004-2012, 2012. Disponível em:< sciencedirect.ez15.periodicos.capes.gov.br/science/article/pii/S0891422212001497?via\%3Dihub>. Acesso em 05 Jan. 2019.

BAUMGARTNER, R.N. Body composition in healthy aging. Annals new york academy of sciences, v. 904, p. 437-448, 2000. Disponível em:< https://nyaspubs.onlinelibrary.wiley.com/doi/epdf/10.1111/j.17496632.2000.tb06498.x>. Acesso em 10 Jan. 2019.

BENTO, Cláudia; MATOS, A.C., CORDEIRO, A., RAMALHO, A. Vitamin A deficiency is associated with body mass index and body adiposity in women with recommended intake of vitamin A. Nutrición Hospitalaria,v.35, n.05, p. 1072-1078, $\quad 2018 . \quad$ Disponível $\quad$ em https://www.nutricionhospitalaria.org/index.php/articles/01630/show\#!>. Acesso em 10 Jan. 2019.

BRAND-WILLIAMS, W.; CUVELIER, M. E.; BERSET, C. Use of a free radical method to evaluate antioxidant activity. LWT - Food Science and Technology, v. 28, n. 1, p. 25-30, 1995. Disponível em :< http://radio.cuci.udg.mx/bch/EN/Manuals/Techniques/DPPHoriginal_LebensWissTechnol_1995-v28p25.pdf>. Acesso em 10 Jan. 2019.

BRAVO-JOSÉ, P.; MORENO, E., ESPERT, M., ROMEU, M., MARTÍNEZ, P., NAVARRO, C. Prevalence of sarcopenia and associated factors in institutionalised older adult patients. Clinical Nutrition ESPEN, v. 27, p.113-119, 2018.

CARNEY, Gemma M. Toward a gender politics of aging. Journal of Women \& Aging, v.30, n.3, p.242-258, 2018.

CHUMLEA, William Cameron; ROCHE, Alex F.; STEINBAUGH, Maria L. Estimating stature from knee height for persons 60 to 90 years of age. Journal of the American Geriatrics Society, v.33, p.116-120, 1985.

CHOI, Kyung Mook. Sarcopenia and sarcopenic obesity. The Korean Journal of Internal Medicine, v.31, p.10541060, 2016. Disponível em:<https://www.ncbi.nlm.nih.gov/pmc/articles/PMC5094937/>. Acesso em: 20 Jan. 2019.

CRUZ-JENTOFT, Alfonso J.; BAEYENS, J.P., BAUER, J.M., BOIRIE, Y., CEDERHOLM, T., LANDI, F., MARTIN, F.C., MICHEL, J.P., ROLLAND, Y., SCHNEIDER, S.M., TOPINKOVÁ, E., VANDEWOUDE, M., ZAMBONI, M. Sarcopenia: European consensus on definition and diagnosis: report of the European Working Group on sarcopenia in older people. Age Ageing, v.39, $\quad$ n.4, $2010 . \quad$ Disponível em:<https://www.ncbi.nlm.nih.gov/pmc/articles/PMC2886201/>. Acesso em: 05 de Fev. 2019.

CRUZ-JENTOFT, Alfonso J.; BAHAT, G.; BAUER, J.; BOIRIE, Y.; BRUYÈRE,O.; CEDERHOLM, T.; COOPER, C.; LANDI, F.; ROLLAND, Y.; SAYER, A.A.; SCHNEIDER, S.M.; SIEBER, C.C., TOPINKOVA, E.;VANDEWOUDE, M.;VISSER, M.; ZAMBONI, M. Sarcopenia: revised European consensus on definition and diagnosis. Age and Ageing, v.48, p. 16-31, 2019. Disponível em:<https://www.ncbi.nlm.nih.gov/pmc/articles/PMC6322506/>. Acesso em 05 Fev. 2019.

DESROSIERS, Johanne; , BRAVO, G.; HÉBERT, R.; DUTIL, E. Normative data for grip etrength of elderly men and women. American Journal of Occupational Therapy, v.49, n.7, p.637-44, 1995.

DODDS, Richard M.; DODDS, R.M.; SYDDALL, H.E.; COOPER, R.; BENZEVAL, M.; DEARY, I.J.; DENNISON, E.M.; DER, G.; GALE, C.R.; INSKIP, H.M.; JAGGER, C.; KIRKWOOD, T.B.; LAWLOR, D.A.; ROBINSON, S.M.; STARR, J.M.; STEPTOE, A.; TILLING, K.; KUH, D.; COOPER C.; SAYER, A.A. Grip strength across the life course: normative data from tw elve 
British studies. PLoS One, v. 09, n. 12, p.014, 2014. Disponível em: <https://www.ncbi.nlm.nih.gov/pmc/articles/PMC4256164/>. Acesso em 15 Fev. 2019.

FRIED, Linda F.; BOUDREAU, R.; LEE, JS; CHERTOW, G; KURELLA-TAMURA, M; SHLIPAK, MG; DING, J; SELLMEYER, D; TYLAVSKY, FA; SIMSONICK, E; KRITCHEVSKY, SB; HARRIS, TB; NEWMAN, AB. Kidney function as a predictor of loss of lean mass in older adults: health, aging and body composition study. Journal of the American Geriatrics Society, $\quad$ v. $\quad 55, \quad$ p. $\quad 1578-1584, \quad 2007 . \quad$ Disponível em:<https://onlinelibrary.wiley.com/doi/epdf/10.1111/i.1532-5415.2007.01398.x>. Acesso em 15 Fev. 2019.

FURR, H. C.; TANUMIHARDJO, O.; OLSON, J. A. Training manual for assessing vitamin A status by use of the modified relative dose response and the relative dose response assays Sponsede by the USAID vitami $n$ A. Field Support Project-Vital, Washington, pp. 70, 1992.

HSU, Kuo-Jen; LIAO, Chun-De; TSAI, Mei-Wun; CHEN, Chiao-Nan. Effects of Exercise and Nutritional Intervention on Body Composition, Metabolic Health, and Physical Performance in Adults with Sarcopenic Obesity: A MetaAnalysis. Nutrients, v.11, n. 2163, p.1-15, 2019. Disponível em:< https://www.ncbi.nlm.nih.gov/pmc/articles/PMC6770949/>. Acesso em 15 Fev. 2019.

IVACG (International Vitamin A Consultative Group). Improving the vitamin A status of populations. USAID-ILSI. 2003.

JACKSON, Andrew S.; POLLOCK, Michael L. Generalized equations for predicting body density of men. British Journal of Nutrition, v. 40, p. 497-504, 1978.

JACKSON, Andrew S.; POLLOCK, Michael L..; WARD, Ann. Generalized equations for predicting body density of women. Medicine \& Science in Sports \& Exercise, v.12, p. 175-82, 1980.

KIM, H; HIRANO, H; EDAHIRO, A; OHARA, Y; WATANABE, Y; KOJIMA, N; KIM, M; HOSOI, E; YOSHIDA, Y; YOSHIDA, H; SHINKAI, S. Sarcopenia: Prevalence and associated factors based on different suggested definitions in community-dwelling older adults. Geriatrics Gerontology International, v.16, p.110-122, 2016. Disponível em:https://onlinelibrary.wiley.com/doi/epdf/10.1111/ggi.12723. Acesso em 05 Mar. 2019.

LAMEU, Edson Braga; GERUDE, Mauricio Freitas; CORRÊA, Regina Célia; LIMA, Keite Azevedo. Adductor policis muscle: a new anthropometric parameter. Revista do Hospital das Clíni cas, v. 59, n.2, p.57-62, 2004.

LANDI, F; LIPEROTI, R; FUSCO, D; MASTROPAOLO, S; QUATTROCIOCCHI, D; PROIA, A; RUSSO, A; BERNABEI, R; ONDER, G. Prevalence and Risk Factors of Sarcopenia Among Nursing Home Older Residents. The Journals of Gerontology, v. 67, n.1, p.48-55, 2012.

LIGUORI, Ilaria; RUSSO, Genaro; ARAN, Luisa; BULLI, Giulia; CURCIO, Francesco; DELLA-MORTE, David; GARGIULO, Gaetano; TESTA, Gianluca; CACCIATORE, Francesco; BONADUCE, Domenico; ABETE, Pasquale. Sarcopenia: assessment of disease burden and strategies to improve outcomes. Clinical Interventions in Aging, v.13, 2018

LOHMAN, Timothy G. Anthropometric Standardization Reference Manual. Champaing, Illinois: Human Kinetics, p.28-80, 1988.

MARTY, Eric; LIU, Yi; SAMUEL, André; OR, Omer; LANE, Joseph. A review of sarcopenia: Enhancing awareness of an increasinglyprevalent disease. Bone, v.105, p.276-286, 2017. Disponível em: <https://reader.elsevier.com/reader/sd/pii/s8756328217303459?token=14AF8E9A308A67420F68DA3FF5A0FA9 E7BB2D85C571BA6E15FA34DCAA852D5DC83329D6C9E7AED68580CAE2189CAF472>. Acesso em 23 Maio 2019.

MARZETTI, E; CALVANI, R; TOSATO, M; CESARI, M; DI BARI, M; CHERUBINI, A; COLLAMATI, A; D'ANGELO, E; PAHOR, M; BERNABEI, R; LANDI, F. Sarcopenia: an overview. Aging clini cal and experimental research, v. 29, p. 11-17, 2017. Disponível em: <https://link.springer.com/content/pdf/10.1007\%2Fs40520-016-0704-5.pdf>. Acesso em 05 abr. 2019.

MEDRONHO, Roberto A. Epidemiologia. 2 ed. São Paulo: Atheneu, 2009. 
MESQUITA, Alice Ferreira; SILVA, Emanuelle Cruz da; EICKEMBERG, Michaela; RORIZ, Anna Karla Carneiro; BARRETO-MEDEIROS, Jairza Maria; RAMOS, Lílian Barbosa. Factors associated with sarcopenia in institutionalized elderly. Nutrición Hospitalaria, v.34, n.2, p.345-351, 2017. Disponível em: < https://pubmed.ncbi.nlm.nih.gov/28421788/?from_term=\%28sarcopenia\%29+AND+\%28institucionalized+elder ly\%29\&from_filter=years.20152020\&from_exact_term=\%28sarcopenia\%29+AND+\%28institutionalized+elderly\%29\&from_pos=3>. Acesso em 28 maio 2020.

MILAGRES, Luana Cupertino; MARTINHO, Karina Oliveira; MILAGRES, Diana Cupertino; FRANCO, Fernanda Silva; RIBEIRO, Andréia Queiroga; NOVAES, Juliana Farias de. Relação cintura/estatura e índice de conicidade estão associados a fatores de risco cardiometabólico em idosos. Ciência \& Saúde Coletiva, v.24, n.4, p. 1451-1461, 2019. Disponível em: < https://www.scielo.br/scielo.php?script=sci_arttext\&pid=S1413-81232019000401451 Acesso em 29 maio 2020.

MINAYO, Maria Cecília de Souza; FIRMO, Joselia Oliveira Araujo. Longevity: bonus or onus? Ciência \& Saúde Coletiva, v.24, n.1, 2019. Disponível em: <http://www.scielo.br/pdf/csc/v24n1/1678-4561-csc-24-01-0004.pdf>. Acesso em 20 abr. 2019.

MUSSOI, Thiago Duran. Avaliação nutricional na prática clínica: da gestação ao envelhecimento. Rio de Janeiro: Guanabara Koogan, 2015.

NAKANO, Márcia Mariko. Versão brasileira da Short Physical Performance Batery-SPPB: adaptação cultural e estudo da confiabilidade. 2007. 181 f. Dissertação (Mestrado em Educação) - Faculdade de Educação, Universidade Estadual de Campinas, São Paulo. Disponível em:< http://repositorio.unicamp.br/bitstream/REPOSIP/252485/1/Nakano_MarciaMariko_M.pdf>. Acesso em 20 abr. 2019.

Nutrition Screening Initiative. Incorporating nutrition screening and interventions into medical practice. A monograph for physicians. Washington, D.C. US: American Academy of Family Physicians. The American Dietetic Association. National Council on Aging Inc; 1994.

OHKAWA, H.; OHISHI, N.; YAGI, K. Assay for lipid peroxides in animal tissues by thiobarbituric acid reaction.

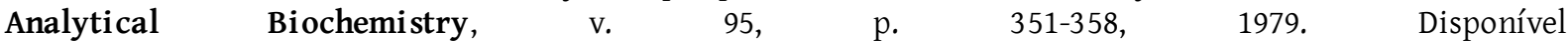
em:<https://reader.elsevier.com/reader/sd/pii/0003269779907383?token=51FE1208215CC6EEB49FC9EDC629A BBD64707581B5897BD405C7740B81732DCAC1DC20484D73CAB7962EAE2A55914321>. Acesso em 20 abr. 2019.

OLAWUYI, Abisola T; ADEOYE, , Ikeola A. The prevalence and associated factors of non-communicable disease risk factors among civil servants in Ibadan, Nigeria. PLOS ONE, v.13, n. 9, p.1-19, 2018. Disponivel em:https://www.ncbi.nlm.nih.gov/pmc/articles/PMC6136760/. Acesso em 25 abr. 2019.

OLIVEIRA, José Egídio Paulo de; MONTENEGRO JÚNIOR, Renan Magalhães; VENCIO, Sérgio. Diretriz da Sociedade Brasileira de Diabetes - 2017/2018. São Paulo: Clannad, 2017.

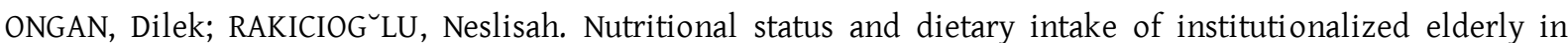
Turkey: A cross-sectional, multi-center, country representative study. Archives of Gerontology and

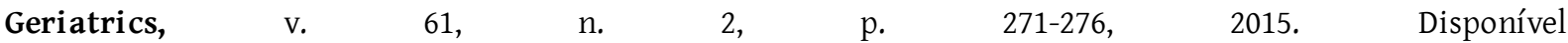
em:<https://reader.elsevier.com/reader/sd/pii/S0167494315000734?token=05F7AD2075DF0740727F8EBB 709F5 569DBA410B58D4F4315FA4656B0444DAEA40D38CE748B6842B504ADB417775C96B7>. Acesso em 02 maio 2019.

PINHEIRO, Natália Cristina Garcia; HOLANDA, Vinícius Carlos Duarte; MELO, Laércio Almeida; MEDEIROS, Annie Karoline Bezerra de Medeiros; LIMA, Kenio Costa. Inequality in the characteristics of the institutionalized elderly in the city of Natal, Brazil. Ciência \& Saúde Coletiva, v. 21, n. 11, p. 3399-3405, 2016. Disponível em:<http://www.scielo.br/pdf/csc/v21n11/1413-8123-csc-21-11-3399.pdf>. Acesso em 05 maio 2019.

PINHEIRO, PA; COQUEIRO, RDS; CARNEIRO, JAO; CORREIA, TML; PEREIRA, R; FERNANDES, MH. Anthropometric indicators as screening tools for sarcopenia in older adult women. Enfermería Clínica, n.19, 2019. 
RODRÍGUEZ-REJÓN; RUIZ-LÓPEZ; ARTACHO. Diagnóstico y prevalencia de sarcopenia en residencias de mayores: EWGSOP2 frente al EWGSOP1. Nutrición Hospitalaria, v.36,n.5, p.1074-1080, 2019.

Rodríguez-Rejón AI, Ruiz-López MD, Wanden-Berghe C, Artacho R. Prevalence and diagnosis of sarcopenia in residential facilities: a systematic review. Advances in Nutrition, v.10, n.1, p. 51-58, 2019.

ROSA, Tábada Samantha Marques; MORAES, Anaelena Bragança de; FILHA, Valdete Alves Valentins dos Santos. The institutionalized elderly: sociodemographic and clinical-functional profiles related to dizziness. Brazilian Journal of Otorhinolaryngology, v.82, n.2, p.159-169, $2016 . \quad$ Disponível em:<http://www.scielo.br/pdf/bjorl/v82n2/pt_1808-8686-bjorl-82-02-00159.pdf. Acesso em 15 maio 2019.

ROSENBERG, I. Summary comments: epidemiological and methodological problems in determining nutritional status of older persons. The American Journal of Clinical Nutrition, v.50, n. 05, p. 1231-1233, 1989.

SANTOS, Vanessa Ribeiro dos, CHRISTOFARO, Diego Giulliano Destro, GOMES, AGOSTINETE, Igor Conterato, , FREITAS JÚNIOR, Ricardo Ribeiro, , FORTE, Ismael. Factors associated with sarcopenia in subjects aged 80 years and over. Revista de Nutrição, v. 28, n. 3, p. 319-326, 2015. Disponível em <http://www.scielo.br/pdf/rn/v28n3/1415-5273-rn-28-03-00319.pdf>. Acesso em 30 maio 2019.

SHAW, S.C.; DENISON, E.M.; COOPER, C. Epidemiology of Sarcopenia: Determinants Throughout the Lifecourse. Nature Reviews Rheumatology, v.13, n.6, p.340-347, 2017. Disponível em:< https://www.ncbi.nlm.nih.gov/pmc/articles/PMC5444517/>. Acesso em 22 maio 2020.

SIEBER, Cornel C. Malnutrition and Sarcopenia. Aging Clinical and Experimental Research, v.31, p.793-798, 2019. Disponível em:< https://link.springer.com/content/pdf/10.1007/s40520-019-01170-1.pdf>. Acesso em 22 maio 2020.

SOARES, Antônio Vinícius; MARCELINO, Elessandra; MAIA, Késsia Cristina; JÚNIOR, Noé Gomes Borges. Relation Between Functional Mobility and Dynapenia in Institutionalized Frail Elderly. Einstein, v.15, n.3, p.278-282, 2017. Disponível em:< https://www.ncbi.nlm.nih.gov/pmc/articles/PMC5823040/>. Acesso em 22 maio 2020.

STUDENSKI, Stephanie A; PETERS, Katherine W; ALLEY, Dawn E; CAWTHON, Peggy M; MCLEAN, Robert R; HARRIS, Tamara B.; FERRUCCI, Luigi; GURALNIK, Jack M.; FRAGALA, Maren S; KENNY, Anne M; KIEL, Douglas P; KRITCHEVSKY, Stephen B; SHARDELL, Michelle D; DAM, Thuy-Tien L; VASSILEVA, Maria T. The FNIH sarcopenia project: rationale, study description, conference recommendations, and final estimates. The journals of gerontology, $\quad$ v. $69, \quad$ p. 547-558, 2014. $\quad$ Disponível em:< https://www.ncbi.nlm.nih.gov/pmc/articles/PMC3991146/>. Acesso em 15 jun. 2019.

TAWFIK, Heba M. Waist height ratio and waist circumference in relation to hypertension, Framingham risk score in hospitalized elderly Egyptians. Egypt Heart Journal, v.70, n.3, p.213-216, 2018.

TOSATO, M; MARZETTI, E; CESARI, M; SAVERA, G; MILLER, RR; BERNABEI, R; LANDI, F; CALVANI, R.Measurement of muscle mass in sarcopenia: from imaging to biochemical markers. Aging Clinical and Experimental Research, v. 29, p. 19-27, 2017. Disponível em:<https://link.springer.com/content/pdf/10.1007\%2Fs40520-0160717-0.pdf>. Acesso em 20 jun. 2019.

TRIANA, Federico Cuesta. Valoración de sarcopenia: de la investigación hacia la práctica clínica. Nutrición Hospitalaria, v. 36, n. 05, p. 999-1000, 2019.

VELLAS, B; GUIGOZ, Y; GARRY, PJ; NOURHASHEMI, F; BENNAHUM, D; LAUQUE, S; ALBAREDE, JL. The Mini Nutritional Assessment (MNA) and its use in grading the nutritional state of elderly patients. Nutrition, p.1559, 1999.

VLIETSTRA, Lara; STEB BINGS, Simon; MEREDITH-JONES, Kim; ABBOTT, J. Haxby; TREHARNE, Gareth J.; WATERS, Debra L. Sarcopenia in osteoarthritis and rheumatoid arthritis: The association with self-reported fatigue, physical function and obesity. PLoS One, v14, n.6, 2019. Disponível em:< https://www.ncbi.nlm.nih.gov/pmc/articles/PMC6553728/>. Acesso em 26 maio. 2020. 
WELCH, A.A.;JENNINGS, A.; KELAIDITI, E.; SKINNER, J.; STEVES, C.J. Cross-Sectional Associations Between Dietary Antioxidant Vitamins C, E and Carotenoid Intakes and Sarcopenic Indices in Women Aged 18-79 Years. Calcifed Tissue International, v. 106, p.331-342, 2020. Disponível em:< https://www.ncbi.nlm.nih.gov/pmc/articles/PMC7072069/>. Acesso em 27 maio. 2020.

WHO. Indicators for assessing vitamin A deficiency and their application inmonitoring and evaluating intervention programs. Geneva, 1996.WHO/NUT/96.10

WHO. Obesity: preventing and managing the global epidemic of obesity. Report of the Who Consultation of Obesity. Geneva, 1997.

\section{CONTRIBUIÇÃO DOS AUTORES:}

Janilson Avelino da Silva

Autor principal e participou de todas as etapas do estudo.

Aléssio Tony Cavalcanti de Almeida

Concepção do projeto e auxílio no cálculo do número amostral e análise estatística. Escrita do artigo.

\section{Ithaissa Lacerda Tavares}

Coleta de dados e elaboração do artigo.

Keyth Sulamitta de Lima Guimarães

Coleta de dados e elaboração do artigo.

\section{Maria José de Carvalho Costa}

Concepção do projeto e auxílio nas metodologias do estudo. Escrita do artigo.

\section{Renata Leite Tavares}

Coleta de dados e elaboração do artigo.

\section{Sandyerlly da Paz do Nascimento}

Cleta de dados e elaboração do artigo.

Maria da Concei ção Rodrigues Gonçalves

Concepção do projeto, auxílio nas metodologias do estudo. Análise dos resultados. Escrita do artigo.

\section{$(\mathrm{cc})$ Br}

Este trabalho está licenciado com uma Licença Creative Commons - Atribuição 4.0 Internacional. 